August 12-15, 2012, Chicago, IL, USA

\title{
DETC2012-70383
}

\section{A DESIGN METHOD BASED ON ANT COLONY OPTIMIZATION FOR COMPLIANT MECHANISMS: INTRODUCTION AND APPLICATION TO A SURGICAL TOOL}

\author{
L. Rubbert, P. Renaud and J. Gangloff \\ LSIIT \\ University of Strasbourg - CNRS, France \\ Email: rubbert@unistra.fr \\ pierre.renaud@unistra.fr \\ jacques.gangloff@unistra.fr
}

\begin{abstract}
This paper presents a design method dedicated to compliant mechanisms, with emphasis on the use of ant colony optimization to determine the optimal geometry of a mechanism. Ant colony optimization is of particular interest because it does not need any fine tuning of its internal parameters. This robustness and the efficiency of the design method are assessed in the context of the design of a surgical tool. The method is then used to propose a new architecture for an active cardiac stabilizer with integrated actuation, contrary to existing architectures.
\end{abstract}

\section{INTRODUCTION}

Compliant mechanisms are of great interest for their high level of accuracy and the absence of backlash or friction. Their design remains however delicate, because their analysis requires to take into account the kinematic behavior, the stresses in the mechanism and the mechanism stiffness that limits the achievable displacements. Several methods have been therefore proposed. On the one hand, the synthesis of the compliant mechanism can be performed using topology optimization [1-3]. On the other hand, the mechanism architecture can be selected by considering a rigid-body mechanism in a first step, before an optimization of the corresponding compliant mechanism using a pseudo-rigid body model (PRBM [4]) [4-11]. This approach appeared often an efficient design strategy that we thus consider in this paper.
The ability to integrate sensing and actuation functions as well as to manufacture the mechanism are all the more important as the mechanism size is reduced. These aspects can be included as design constraints during an optimization. The formulation of the optimization problem includes then however a large number of non-linear conditions. For such type of optimization problem, gradient-based optimization algorithms are confronted to the existence of many local minima in the optimization function. Metaheuristic optimizations, based on stochastic algorithms, can constitute interesting alternatives. Genetic algorithms, evolutionary algorithms are well known approaches $[12,13]$. Hereafter, we propose to investigate for the optimization the use of an ant colony optimization (ACO). This algorithm is essentially interesting for its ease of implementation: the method does not need a delicate tuning of its internal parameters to be efficient. ACO has been proposed for different optimization problems $[14,15]$ and we recently proposed its use for the optimization of a compliant mechanism [10].

In this paper, we extend the work presented in [10] and introduce two main contributions. First, a design method based on the use of ACO with PRBM is presented and evaluated. The evaluation is performed in the context of the design of a surgical tool, with an experimental analysis of a proof of concept. Second, in the context of active cardiac stabilization, the design and optimization of a new compliant mechanism is presented. Contrary to previously proposed devices, the actuation is fully integrated in the body of the tool.

This paper is organized as follows. In section 2, the design 


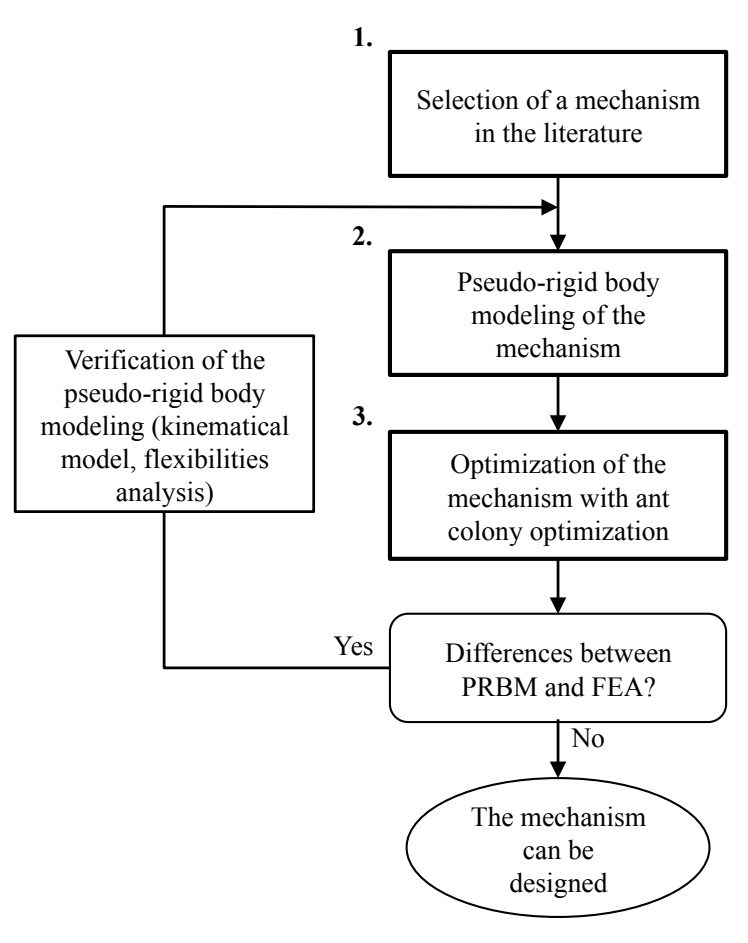

FIGURE 1. The design method considered for the design of a compliant mechanism.

method we propose is presented and the principle of ant colony optimization adapted to our method is developed. In section 3, the method is detailed and evaluated experimentally with the design of a 1-DOF mechanism in the context of an active surgical tool. The optimization results for an active surgical tool are then investigated in section 4 before concluding on the interest of the design method and future developments.

\section{DESIGN METHOD}

\subsection{The method}

The design method is composed of three main steps (Fig. 1). As mentioned in the introduction, we here consider the use of PRBM to model compliant mechanisms. Each flexure hinge of a compliant mechanism is hence considered as a joint with a nonnull stiffness, and the links connecting them as rigid parts. We can therefore take advantage of the analysis tools for rigid-body mechanisms to select and model the architecture of compliant mechanisms.

Step 1: Selection of a mechanism In the first step, a rigid-body mechanism is selected in the literature. A mechanism is selected if it is compatible with the five following selection criteria:
1. The mechanism must exhibit the degrees of freedom required by the application.

2. Among mechanisms with the same degrees of freedom, the selected mechanism must have the smallest number of joints. Minimizing the number of joint allows us to design stiffer links for a given volume. The accuracy of PRBM, that will not take into account any distributed flexibilities in the links, will hence be increased.

3. The mechanism configuration must be compatible with the prescribed shape.

4. The architecture of the mechanism must allow the integration of the actuators and the sensors.

5. The manufacturing of the compliant mechanism must appear achievable.

If several mechanisms seem compatible with the application, they are considered in the steps 2 and 3 , and their relative performance are compared after optimization.

Step 2: Modeling of the mechanism From the knowledge of the mechanism geometry, the kinematic model can be derived to relate the input and output displacements. Since the modeling of the mechanism is performed with PRBM, the stiffness of the compliant joints can be expressed from the value of the geometrical parameters defining their shape $[16,17]$. The global stiffness of the mechanism can then be established using a static analysis. Finally, the mechanical stresses in each joint can be obtained based on the static analysis and the geometry of the joints.

At the end of the second step, it is therefore possible to compute the performance of a mechanism for a given set of geometrical parameters $\xi$ describing the mechanism dimensions.

Step 3: Optimization of the mechanism The design problem is expressed as an optimization problem with an objective function $f$ to be maximized and a set of constraints $\Omega$ related to the integration and manufacturing of the mechanism. The parameter set $\xi$ is optimized using an ant colony optimization method detailed in the next section. The search space $S$ contains all geometrical parameters sets $\xi$ that are defined in continuous ranges. The objective function is the formulation of the mechanism performance when $\Omega$ is respected.

Validation The performance of the optimized mechanism is compared to the one estimated from finite element analysis (FEA). If significant errors between the PRBM and FEA predictions appear, the kinematic model must be first verified: for a given input displacement, the same output displacement must be obtained with FEA and PRBM. If the kinematic model is correct, the PRBM needs to be improved by taking into account distributed flexibilities that appear as significant. 


\subsection{ANT COLONY OPTIMIZATION}

Ant colony optimization for compliant mechanisms

Ant Colony Optimization (ACO) is a stochastic optimization method. It mimics the behavior of ants that are able to optimize collectively the path between their house and a source of food by using a chemical marker, called pheromone, for local stigmergic communication. The colony evolves by sequences of local moves to find the best path, here a parameter set $\xi$ describing the mechanism, and the progression is based on local information without lookahead. Considered initially for combinatorial problems [18], it has been extended recently to continuous domain $[19,20]$, which is our case.

An adaptation of the ACO algorithm is proposed here. In fact, in [19], different paths are explored simultaneously and cross-overs between these paths are allowed. In our case, as the optimization problem is very constrained, cross-overs between different solutions will decrease the efficiency of the algorithm. Thus, instead of making cross-overs among parameters of different initial solutions as in [19], we propose to explore sequentially one solution after another. As a consequence, the algorithm explores the neighborhoods of the initial solution as real ants would do. The pheromone used for local communication by the ants is first simulated by the exploration of a restrained domain and its evaporation is simulated by the abandon of the less efficient solutions. Then, to improve the search of the optimum, the pheromone is simulated with the use of a gaussian distribution law around the best solution.

The algorithm The ACO is composed of four main steps and is illustrated, in Fig. 2, with an example based on a twoparameter optimization problem.

In the first step, to start the algorithm, geometrical parameter sets $\xi$ are generated randomly, using a uniform distribution law in all the parameter domain $S$. The random search is repeated until at least one solution respecting all the constraints is found.

In the second step, for each parameter set $\xi$ that respects all the constraints $\Omega$ (in grey on Fig. 2), new parameter sets $\xi$ are generated with a uniform distribution law in a restrained domain surrounding the best found solution, to improve the solution performance. To rank the solutions, each mechanism that respects all the constraints is tested and associated to its performance value. Non-viable mechanisms are associated to a null value. To avoid a blockage of the solution evolution, the size of the restrained domain is increased. If no more better solution is found at this point the second step is stopped. The algorithm is quite robust with the thresholds used to restrain or widen the search space: their values can be easily set and not strongly affect the success of the optimization. This will be illustrated in the following section during the evaluation of the method.

In the third step, the ants collaboration is simulated to find the best solution. The solution generation is performed by switching to a gaussian distribution law for the look-around radius of each parameter. The mean value and standard deviation are initiated using the sets of viable configurations determined in the second step and then computed with those found in this step. The ranking of the solutions is achieved similarly to the one performed in the previous step. The gaussian law helps to increase the density of parameter sets around the best solution and therefore allows us to decrease the number of parameter sets to generate, and finally to improve the efficiency of the algorithm.

The standard deviation of each parameter tends to zero during the third step. When all these values become close to zero, local search using a gradient based method is performed to refine locally the solution.

These four steps are repeated until the mechanism performance is reached or the calculation time is overpassed.

\section{EVALUATION OF THE DESIGN METHOD}

In order to evaluate the design method, a proof of concept is designed in the context of a surgical application. In such a context, strong design constraints exist due to the small size of the surgical instruments. It is therefore interesting to assess the design method within this context.

\subsection{CONTEXT}

Active cardiac stabilizers [21,22] have been proposed to immobilize the surface of a beating heart during surgery. These devices are mainly composed of a shaft (Fig. 3) whose tip is related to the beating heart surface. For previously proposed devices, the active structure, composed of a compliant mechanism and a PZT actuator, is located at the other tip of the shaft to cancel any displacement measured by high-speed vision of the beating heart surface due to the forces applied by the heart. Previous experiments have shown that the stabilizer tip must exhibit displacements in the two directions perpendicular to the shaft. The displacement must reach $1 \mathrm{~mm}$ to cancel the deflections caused by a heart force of $5 \mathrm{~N}$.

In order to increase the device compactness, we consider here the integration of the actuator and the compliant mechanism directly inside the device shaft. The design of the compliant mechanism is strongly constrained by the geometry of the shaft. For the final application, the shaft diameter cannot exceed $12 \mathrm{~mm}$. For the proof of concept dedicated to the evaluation of the design method, we consider a scale factor of 1.5 in order to simplify the experimentation evaluation.

A simple way to integrate the actuator is to consider a piezoelectric stack of cylindrical shape, which axis is parallel to the shaft axis. An actuator compatible with the size constraints provides a displacement in the order of 50 microns. As a consequence, we need to design a compliant mechanism that converts the displacement along the shaft axis into a displacement perpen- 


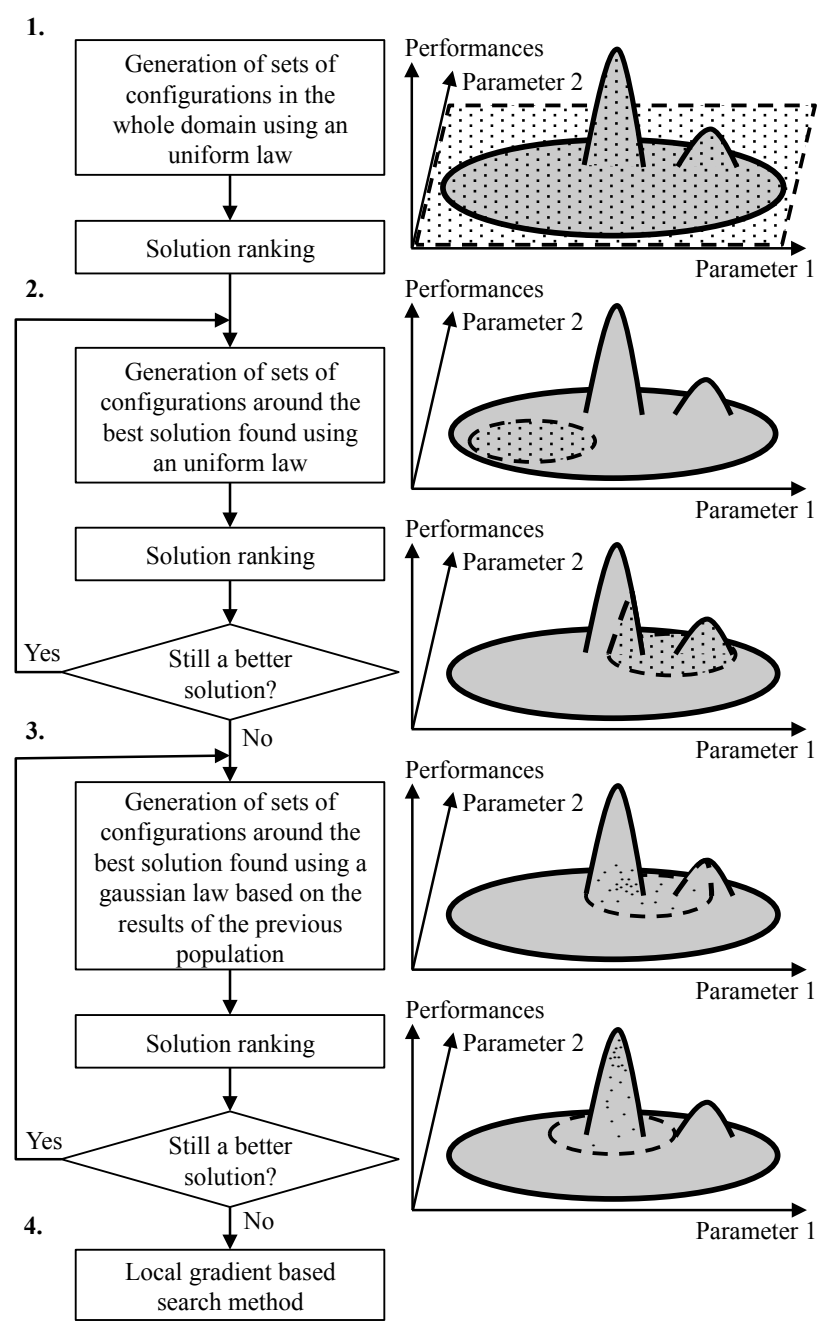

FIGURE 2. Algorithm structure with a simplified illustration of a two-parameter problem for each step.

dicular to the shaft. In addition it must amplify this displacement so that the amplitude of the stabilizer tip displacement is in the order of $1 \mathrm{~mm}$ for a cardiac force of $5 \mathrm{~N}$.

\subsection{SELECTION OF THE MECHANISM}

The selection of a mechanism architecture is guided by the selection criteria presented in section 2 . In this particular context, the mechanism must be of oblong geometry to fit in a tube.

Lever based mechanisms are the simplest amplification mechanisms which provide a linear amplification, i.e. a constant amplification factor but have too limited amplification ratios for our context $[23,24]$. High amplification ratios can be obtained using non-linear effects such as beam buckling [25-27]. Geometrical non-linearities of mechanisms can also be used to

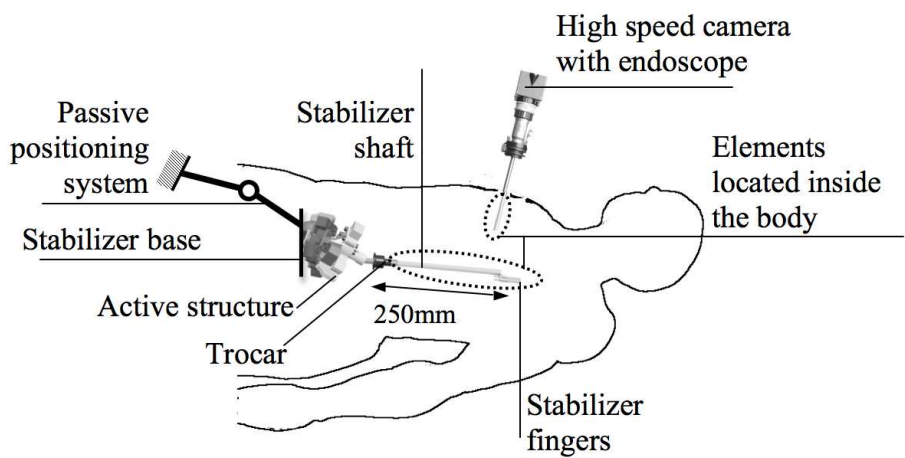

FIGURE 3. Principle of active cardiac stabilization [11].

create so called non-linear amplification mechanisms. Interesting compliant mechanisms have been proposed based on equivalent 4-bar linkages [28-30], five-bar bridge mechanisms [31,32], and combinations of those mechanisms for instance with a symmetrical replication [27,33-35] or assembly of several architectures $[26,32]$.

In our context, two mechanisms are of particular interest. The Scott-Russell mechanism [36] has an overall oblong shape that tends to be compatible with an integration in the stabilizer tubular shaft, and it converts an input displacement in a displacement perpendicular to the main axis of the mechanism. And the 4-bar mechanisms may also be designed to fit in the stabilizer shaft.

Fig. 4a shows how the Scott-Russell mechanism can be integrated in the shaft. Originally, a prismatic joint is included at the input of the mechanism. In order to reduce the number of joints, it is here replaced with a lever mechanism instead of a 4-bar mechanism, which is kinematically equivalent for small displacements. Fig. $4 \mathrm{~b}$ shows the integration of a 4-bar mechanism, with the piezoelectric actuator located inside a tubular shaft and describes schematically the shape of the elements that would compose both mechanisms.

Both mechanisms are composed of revolute joints and their number is identical. In case of the Scott-Russell mechanism, the presence of slender elements can be difficultly avoided in the configuration presented in Fig. 4a due to the link organization. These slender bars may lack of stiffness and may be difficult to machine. In case of the 4-bar mechanism, a better material distribution can be expected due to a better bar arrangement. Moreover, in the Scott-Russell mechanism, the force delivered by the actuator in this configuration tends to produce buckling effects in the bars. In case of the 4-bar mechanism, the force delivered by the actuator introduces tensile stresses in the mechanism bars, without any risk of buckling. The 4-bar mechanism is therefore chosen. 

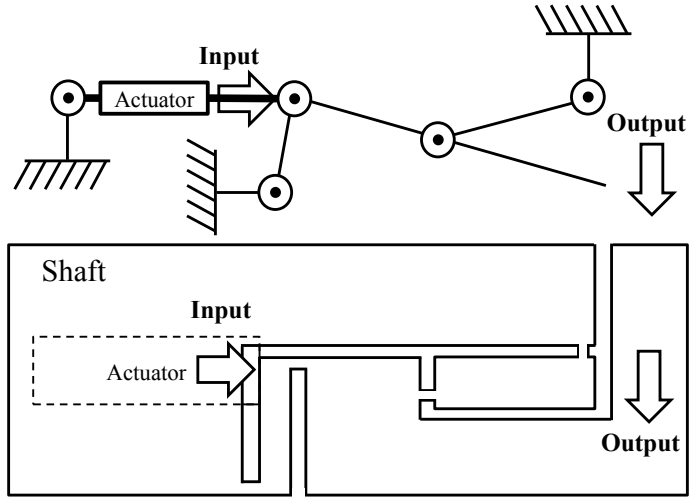

(a) Side view of the shaft with a possible integration of the Scott-Russell mechanism and its actuator.

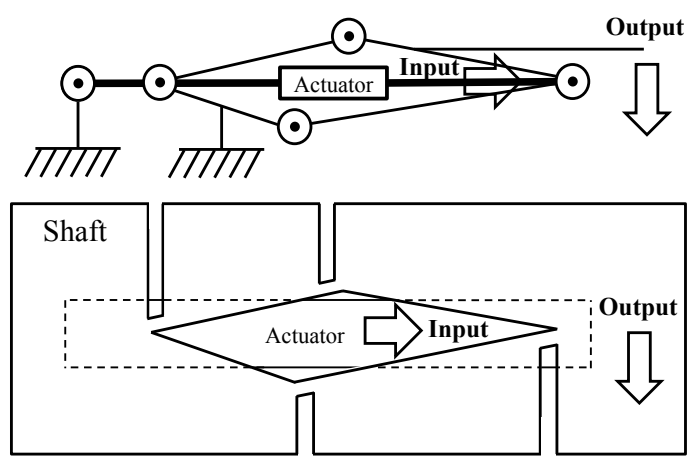

(b) Side view of the shaft with a possible integration of the 4-bar mechanism and its actuator.

FIGURE 4. Kinematic schemes of the two considered mechanisms. The dotted lines represent a possible outer profile of the bars in the compliant structures.

\subsection{Modeling}

The model is represented in Fig. 5a. Six geometrical parameters $\left(L_{0}, L_{1}, L_{2}, L_{3}, L_{4}\right.$ and $\left.L_{5}\right)$ define the mechanism and its initial configuration. Since the mechanism is integrated in a tube, its outer radius $R$ and its thickness $T$ are also needed to define the geometry (Fig. 5b). Circular notch joints are considered that are described by their minimum thickness $t$, the radius $r$ of their circular profile and their width, defined by their position with respect to the tube axis. In fact, the joints in $A$ and $C$ have a width $w_{A}$ and $w_{C}$ depending on $H_{A}$ and $H_{C}$, the distances of their centers to the shaft axis (Fig. 5b), which also depend on the parameters $L_{0}, L_{1}, L_{2}, L_{3}$ and $L_{5}$. The width $w_{B}$ and $w_{D}$ are considered equal to the tube thickness $T$.

If the distances $H$ of the points $A$ or $C$ to the shaft axis re-

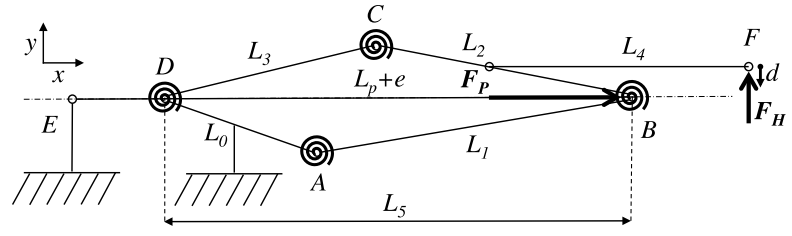

(a) Parameterization of the PRBM of the mechanism.
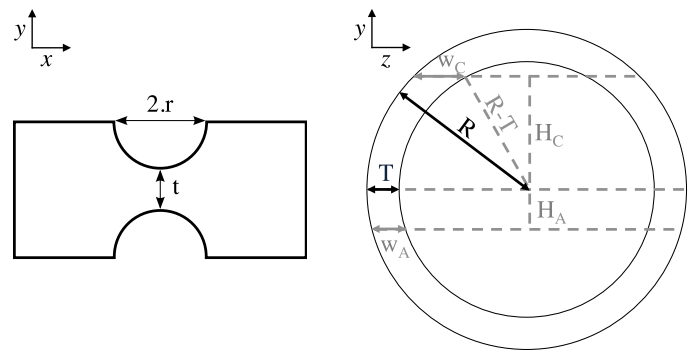

(b) Parameterization of the notch joints.

FIGURE 5. Parameterization of the PRBM of the mechanism and its joints.

spect $H \leq R-T$,

$$
\begin{aligned}
& w_{A}=\sqrt{R^{2}-H_{A}^{2}}-\sqrt{(R-T)^{2}-H_{A}^{2}} \\
& w_{C}=\sqrt{R^{2}-H_{C}^{2}}-\sqrt{(R-T)^{2}-H_{C}^{2}}
\end{aligned}
$$

else

$$
\begin{aligned}
& w_{A}=\sqrt{R^{2}-H_{A}^{2}} \\
& w_{C}=\sqrt{R^{2}-H_{C}^{2}}
\end{aligned}
$$

The relationship between the actuator displacement $e$ and the output displacement $d$ can be easily obtained by expressing the loop closure equations of the loop $A B E$ and $A B C D$, to obtain the position of the points $B$ and $C$ in their initial and final configuration and finally the positions of point $F$ in function of the displacement $e$.

To obtain the achievable output displacement $d$, a static model is derived, since the piezoelectric actuator maximum elongation $e$ depends on the stiffness of the mechanism acting against it. The compliant joint rotational stiffnesses are described using the model derived by Schotborgh et al. [37], described as the most accurate and having the widest range of validity by Yong et al. [17]:

$$
K=\frac{E \cdot w \cdot t^{2}}{12}\left(-0.0089+1.3556 \sqrt{\frac{t}{2 . r}}-0.5227 \frac{t}{2 . r}\right)
$$


with $K$ the joint stiffness, $E$ the Young's modulus, $t, r$ and $w$ the thickness, the radius and the width of the considered joint. The bending moment $M$ in each joint is thus easily obtained:

$$
M=K . \alpha
$$

with $\alpha$ the angular deflection of the joint, computed from the actuator displacement $e$ with the kinematic model.

The stress $\sigma$ in each compliant joint can be expressed analytically by using a beam model, and introducing stress concentration factors refines the stress value [38]:

$$
\sigma=k_{T} \cdot \frac{F}{t \cdot w}+k_{B} \cdot \frac{6 \cdot M}{t^{2} \cdot w}
$$

with $F$ the force normal to the section of the joint, $M$ the bending moment, $k_{T}$ and $k_{B}$ the correction factors for the traction and the bending components.

\subsection{Formulation of the optimization problem}

The output displacement and maximal mechanical stresses in the joints can now be computed for a given parameter set $\xi$. As mentioned in section 2, the optimization problem is defined by a search space $S$, a set of constraints $\Omega$ and an objective function $f$. Additionally, the definition of the restrained domain and its influence mentioned in section 2.2 will be detailed.

The search space $\mathbf{S}$ A path chosen by an ant is a compliant architecture defined by a set $\xi$ of sixteen geometrical parameters: six bar lengths, four joint thicknesses, four radii circular notches, one tube diameter and one tube thickness. The ranges of value of these parameters are limited by the machining performances.

The set of constraints $\Omega$ The ants have to find the best path, i.e. the parameter set $\xi$ that maximizes the output displacement, while respecting a set of constraints:

- The maximum stress in each joint must be compatible with the material properties

- The outer profile of the mechanism must remain inside the tube

- The configuration of the mechanism, that can be defined by the angles between the bars, must remain compatible with the configuration represented in Fig. 4b, for which the mechanism provides an amplification in the correct direction

- The geometry of each joint must not interfere with the shape of the bars as represented in Fig. 4b

- The actuator must fit in the tube
The integration constraints introduce a set of seven linear constraints and fifteen non-linear constraints represented as a set of inequalities. During the optimization, we consider that the actuator is given, since we will use the actuator providing the maximum displacement that can be integrated in the tube, and the tube material properties are known.

The evaluation function $\mathbf{f}$ To evaluate the quality of a path, a performance function $f$ needs to be defined to rank the solutions. For a given actuator the displacement $d$ is increased when the stiffness of the mechanism defined by the joints is decreased. Reducing the stiffness of the joints leads to decrease their cross-sections and thus increase the maximal stress $\sigma$ in the material. It can be interesting to introduce in the performance function the closeness of the maximum stress to the admissible maximum stress for the material. When $\sigma$ is close to the admissible stress the solutions behave like there where only one objective: increasing the displacement $d$. Thus, for a given material, its mechanical properties are used in an optimal way. Hence, the proposed performance function is expressed as:

$$
\operatorname{Performance}(\xi)=\frac{d}{1.01^{\text {abs }\left(\sigma_{\text {objective }}-\sigma_{\max }\right)}}
$$

with $\xi$ the parameter set, $\sigma_{\text {objective }}$ the maximal admissible stress, $\sigma_{\max }$ the maximum stress in the mechanism and 1.01 a penalty factor chosen strictly greater than 1 . Its value depends on the problem: it must be penalizing enough so that solutions with very high or very low maximal stress values are not considered, but it must not be too penalizing to allow some exploration around the maximal admissible stress value. Here the value is chosen so that if the objective displacement is reached and the maximum stress value in the mechanism is close to $2 \%$ of the maximal admissible stress value, $90 \%$ of the objective is reached. In this way, when the stress objective value is almost reached, the only possibility to increase the performance is to maintain the maximal stress value close to this objective value. The ACO does not require any continuity or derivation property for the performance evaluation function. Such a non-linear function can thus be used.

The restrained domain For the second step of the ACO algorithm presented in section 2.2 , we choose a restrained domain corresponding to $20 \%$ of the range of the parameters $\xi$. To avoid a blockage of the solution evolution, we choose that the size of the restrained domain growth incrementally of $4 \%$ until the search space reaches $40 \%$ of the parameter space. The choice of these values do not strongly affect the optimization success: dividing for instance these values by two does not modify the optimization results. 
TABLE 1. OPTIMAL DESIGN PARAMETERS (IN MM) FOR THE PROOF OF CONCEPT

\begin{tabular}{|l|l|l|l|l|l|l|l|}
\hline $\mathrm{L}_{0}$ & $\mathrm{~L}_{1}$ & $\mathrm{~L}_{2}$ & $\mathrm{~L}_{3}$ & $\mathrm{~L}_{4}$ & $\mathrm{~L}_{5}$ & $\mathrm{R}$ & $\mathrm{T}$ \\
\hline 12.26 & 48.50 & 32.44 & 28.10 & 59.54 & 39.90 & 9.00 & 2.00 \\
\hline $\mathrm{t}_{\mathrm{A}}$ & $\mathrm{r}_{\mathrm{A}}$ & $\mathrm{t}_{\mathrm{B}}$ & $\mathrm{r}_{\mathrm{B}}$ & $\mathrm{t}_{\mathrm{C}}$ & $\mathrm{r}_{\mathrm{C}}$ & $\mathrm{t}_{\mathrm{d}}$ & $\mathrm{r}_{\mathrm{d}}$ \\
\hline 0.50 & 1.77 & 1.11 & 1.45 & 0.51 & 1.07 & 0.50 & 2.00 \\
\hline
\end{tabular}

\subsection{EXPERIMENTAL EVALUATION}

A Z30C13 stainless steel tube of $18 \mathrm{~mm}$ diameter is chosen. The yield limit of the material is $500 \mathrm{MPa}$. The chosen piezoelectric actuator (Physik Instrumente P-041.30), compatible with this tube, has a diameter of $12 \mathrm{~mm}$, a length of $80 \mathrm{~mm}$ and can provide a maximum displacement of $45 \mu \mathrm{m}$.

The ACO is implemented using the Matlab software with an Intel Core 2 Duo computer cadenced at $2.26 \mathrm{GHz}$. Even though such an implementation is not optimal, optimization for our problem was achieved in less than six hours with an overall number of performance evaluations in the order of 20 millions. The results of the ACO are given in table 1 .

The performance predicted with the design method is compared with the results of a Finite Element Analysis (FEA), performed using PTC Pro/Mechanica. The displacements of the mechanism tip are compared as well as the maximal stress in the joints. In fact, in the PRBM model, the equilibrium force reached by the actuator, the input displacement and the output displacement are known. The equilibrium force is applied in the FEA and the obtained output displacement is compared to the one of the PRBM. The results of the ACO are in a very good accordance with the FEA: discrepancy in the estimation of the displacement $d$ is below $4 \%$ and below $7 \%$ for the stresses. The device is designed to exhibit a maximal displacement of $0.50 \mathrm{~mm}$.

The prototype is manufactured using CNC machining (Fig. 6). Its performance is evaluated using vision: a visual marker is located at the tip of the prototype, and a high speed camera (Dalsa CAD-6 with a Navitar Precise Eye lens) allows the determination of the marker displacement with an accuracy in the order of $1 \mu \mathrm{m}$ (Fig. 7). The maximum displacement is estimated equal to $0.52 \mathrm{~mm}$ (Fig. 8), within $4 \%$ of the value obtained with the PRBM model as well as with the FEA. Loads have then been applied on the stabilizer tip to evaluate the consistency of PRBM model, FEA and experimental results: for a load up to $5.7 \mathrm{~N}$, above the target value of $5 \mathrm{~N}$, relative errors between the three displacement values are below $5 \%$. Finally, an analysis of the first eigenfrequency has been performed. Its value is around $200 \mathrm{~Hz}$, with $10 \mathrm{~Hz}$ of measurement uncertainty, whereas FEA simulation provides a value of $203 \mathrm{~Hz}$.

Experimental results are very close to those obtained with the numerical simulation and the PRBM. This tends to show

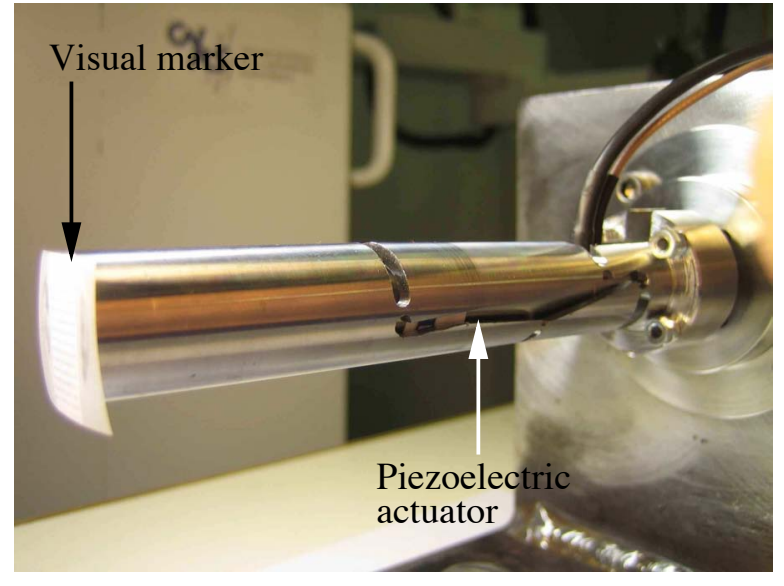

FIGURE 6. Proof of concept with its machined compliant mechanism.

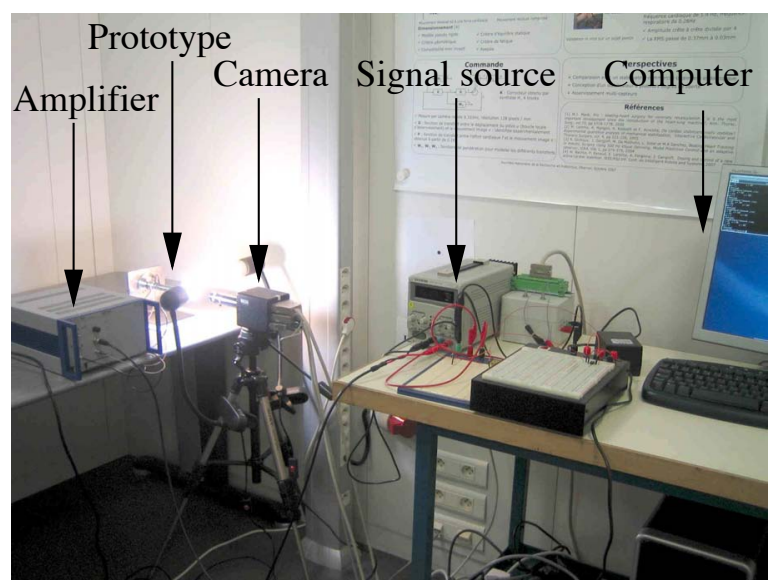

FIGURE 7. Experimental set-up.

that the proposed modeling describes reliably the behavior of the mechanism and can be now applied with enough reliability to the optimization of a compensation mechanism for an active surgical tool composed of a shaft at adequate size.

\section{OPTIMIZATION OF A NEW ACTIVE CARDIAC STA- BILIZER}

In this section, the design method is now applied to define a cardiac stabilizer compatible with the requirements expressed in section 3. The compliant architecture must therefore provide 2 DOF. In vivo experiments have demonstrated [21] that the cardiac forces are not identical in the two directions perpendicular to the stabilizer shaft. The maximum force is equal to $5 \mathrm{~N}$ in one direction and to $1.7 \mathrm{~N}$ in the other direction. These values are considered during the design. 


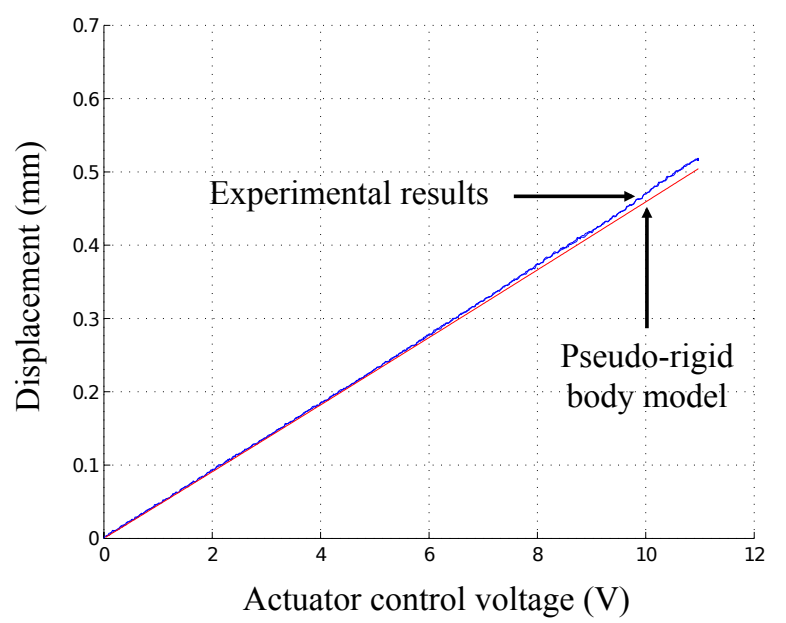

FIGURE 8. Displacements measured using vision and evaluated using the PRBM.

As outlined in section 3, the external diameter of an active cardiac stabilizer cannot exceed $12 \mathrm{~mm}$. PZT actuators compatible with such a diameter provide a maximum displacement in the order of 50 microns for a diameter of $7 \mathrm{~mm}$ and a length of $54 \mathrm{~mm}$.

Parallel architectures usually offer interesting stiffness properties. In our context, the actuators cannot be placed side by side in the shaft. The transmissions between the end-effector, located at the tip of the shaft, and the actuators would then be very long. To limit the device flexibility, a serial architecture is therefore preferred.

The serial mechanism is considered to be the assembly of two 4-bar mechanisms, since the criteria for the mechanism selection are similar to those of the previous section. The optimization of the two mechanisms is performed sequentially. First the mechanism which is the closest to the stabilizer tip is optimized. Then the size of the first compensation mechanism is taken into account in the optimization of the second compensation mechanism.

At the moment, we only consider stresses due to the load in the direction of displacement of each mechanism. The assumption, that remains strong, allows us to explore the potential solutions of fully integrated stabilizers.

\subsection{FIRST COMPENSATION MECHANISM}

In the case of the surgical tool, a $12 \mathrm{~mm}$ diameter shaft in 35NCD16 steel is considered. A $7 \mathrm{~mm}$ diameter piezoelectric actuator from Physik Instrumente (P-007.40, length $54 \mathrm{~mm}$ ) is selected. It has one of the largest available displacement $(60 \mu \mathrm{m})$ for an actuator that can be integrated into the stabilizer shaft. The maximal force delivered by this actuator is $1150 \mathrm{~N}$.
TABLE 2. OPTIMAL DESIGN PARAMETERS (IN MM) FOR THE FIRST MECHANISM

\begin{tabular}{|l|l|l|l|l|l|l|l|}
\hline $\mathrm{L}_{0}$ & $\mathrm{~L}_{1}$ & $\mathrm{~L}_{2}$ & $\mathrm{~L}_{3}$ & $\mathrm{~L}_{4}$ & $\mathrm{~L}_{5}$ & $\mathrm{R}$ & $\mathrm{T}$ \\
\hline 10.22 & 38.60 & 37.76 & 11.19 & 47.32 & 59.15 & 6.00 & 2.00 \\
\hline $\mathrm{t}_{\mathrm{A}}$ & $\mathrm{r}_{\mathrm{A}}$ & $\mathrm{t}_{\mathrm{B}}$ & $\mathrm{r}_{\mathrm{B}}$ & $\mathrm{t}_{\mathrm{C}}$ & $\mathrm{r}_{\mathrm{C}}$ & $\mathrm{t}_{\mathrm{d}}$ & $\mathrm{r}_{\mathrm{d}}$ \\
\hline 0.11 & 0.66 & 0.57 & 1.25 & 0.10 & 0.66 & 0.20 & 2.06 \\
\hline
\end{tabular}

TABLE 3. OPTIMAL DESIGN PARAMETERS (IN MM) FOR THE SECOND MECHANISM

\begin{tabular}{|l|l|l|l|l|l|l|l|}
\hline $\mathrm{L}_{0}$ & $\mathrm{~L}_{1}$ & $\mathrm{~L}_{2}$ & $\mathrm{~L}_{3}$ & $\mathrm{~L}_{4}$ & $\mathrm{~L}_{5}$ & $\mathrm{R}$ & $\mathrm{T}$ \\
\hline 11.93 & 32.10 & 32.98 & 11.43 & 42.64 & 118.00 & 6.00 & 2.00 \\
\hline $\mathrm{t}_{\mathrm{A}}$ & $\mathrm{r}_{\mathrm{A}}$ & $\mathrm{t}_{\mathrm{B}}$ & $\mathrm{r}_{\mathrm{B}}$ & $\mathrm{t}_{\mathrm{C}}$ & $\mathrm{r}_{\mathrm{C}}$ & $\mathrm{t}_{\mathrm{d}}$ & $\mathrm{r}_{\mathrm{d}}$ \\
\hline 0.12 & 0.61 & 0.40 & 1.04 & 0.18 & 0.45 & 0.10 & 0.84 \\
\hline
\end{tabular}

The optimal solution is described in table 2. The first compensation mechanism can exhibit a displacement of $0.9 \mathrm{~mm}$, with maximal stress of $550 \mathrm{MPa}$ for a $5 \mathrm{~N}$ cardiac force. This stress value corresponds to the endurance limit of the material. The performance of the mechanism is confirmed by comparison with FEA results. Relative errors in the estimation of the displacement and the stresses in the compliant joints do not exceed $5 \%$.

\subsection{OPTIMIZATION OF THE SECOND COMPENSA- TION MECHANISM}

The optimal solution is given in table 3 and the device is represented in Fig. 9. The second compensation mechanism can exhibit a displacement of $1.2 \mathrm{~mm}$ under a load of $1.7 \mathrm{~N}$, with a maximal stress of $550 \mathrm{MPa}$. This latter value also corresponds to the endurance limit of the material. The performance of the mechanism is confirmed by comparison with FEA results. Relative errors in the estimation of the displacement and the stresses in the compliant joints do not exceed $3 \%$.

The optimization of each mechanism allow us to fulfill the displacement requirements. However, FEA of the whole device shows that the lateral stiffness of the second mechanism is not satisfactory. A complete modeling, that takes into account the effect of lateral forces is required to deepen the optimization of the device.

\section{CONCLUSION}

In this paper, a design method of compliant mechanism using pseudo-rigid body modeling and an ant colony optimization has been presented. This method has been successfully applied 


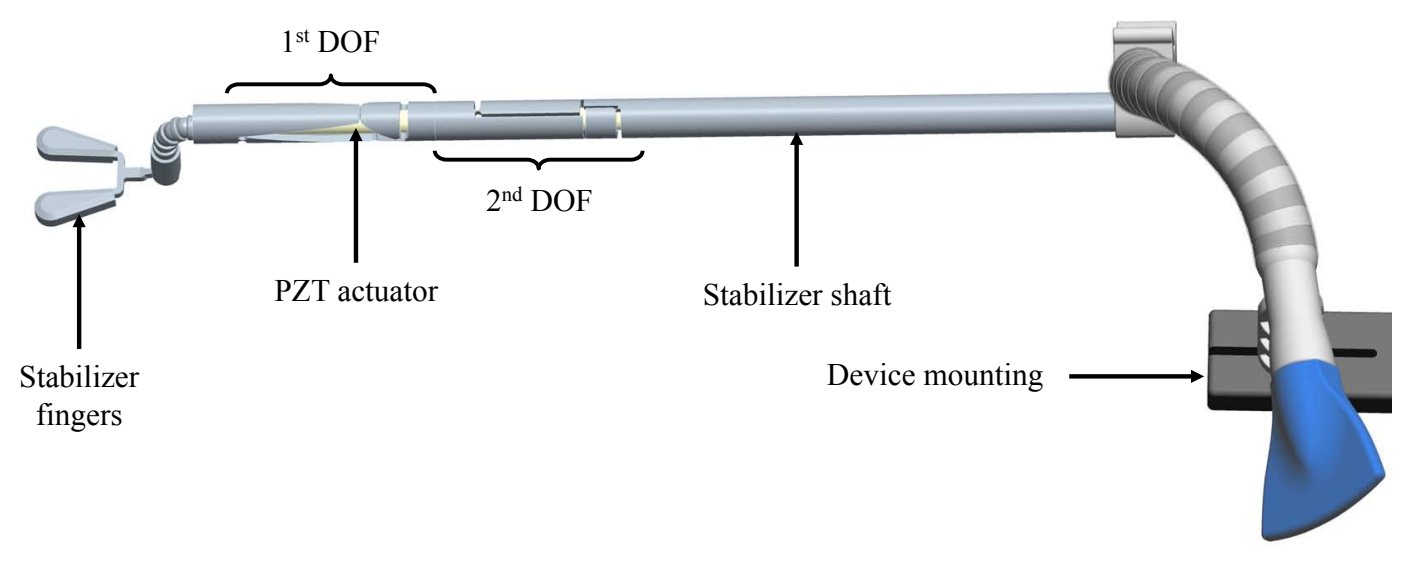

FIGURE 9. Cardiac stabilizer with two integrated compliant mechanisms.

on a proof of concept.

From a medical point of view, active stabilization is a promising approach, and current results on a fully integrated 2DOF compliant mechanism in the stabilizer shaft open new perspectives. Refinement of the modeling is now needed to further investigate the design of the device.

From a compliant mechanism design point of view, using an ant colony optimization algorithm for the optimization of pseudo-rigid body model of compliant mechanisms appears efficient and the algorithm is easy to tune. Further comparison with other optimization techniques will now be performed, and application to other architectures will be considered.

\section{REFERENCES}

[1] Canfield, S., and Frecker, M., 2000. "Topology optimization of compliant mechanical amplifiers for piezoelectric actuators". Structural and Multidisciplinary Optimization, December.

[2] Frecker, M. I., 2003. "Recent advances in optimization of smart structures and actuators". Journal of Intelligent Material Systems and Structures, 14(4-5), pp. 207-216.

[3] Bendsøe, M. P., Lund, E., Olhoff, N., and Sigmund, O., 2005. "Topology optimization - broadening the areas of application". Control and Cybernetics, 34(1), pp. 7-35.

[4] Howell, L., 2001. Compliant mechanisms. Wiley-IEEE.

[5] Murphy, M. D., Midha, A., and Howell, L. L., 1996. "The topological synthesis of compliant mechanisms". Mechanism and Machine Theory, 31(2), pp. 185 - 199.

[6] Baker, M., and Howell, L., 2002. "On-chip actuation of an in-plane compliant bistable micromechanism". Microelectromechanical Systems, Journal of, 11(5), Oct., pp. 566 573.

[7] Luharuka, R., and Hesketh, P. J., 2007. "Design of fully compliant, in-plane rotary, bistable micromechanisms for mems applications". Sensors and Actuators A: Physical, 134(1), pp. 231 - 238. International Mechanical Engineering congress and Exposition 2005 - IMECE 2005, American Society of Mechanical Engineering International Mechanical Engineering Congress and Exposition.

[8] Li, Y., and Xu, Q., 2009. "Design and optimization of an xyz parallel micromanipulator with flexure hinges". Journal of Intelligent \& Robotic Systems, 55, pp. 377-402. 10.1007/s10846-008-9300-z.

[9] Pucheta, M. A., and Cardona, A., 2010. "Design of bistable compliant mechanisms using precisionposition and rigidbody replacement methods". Mechanism and Machine Theory, 45(2), pp. $304-326$.

[10] Rubbert, L., Renaud, P., Bachta, W., and Gangloff, J., 2011. "Compliant mechanisms for an active cardiac stabilizer: lessons and new requirements in the design of a novel surgical tool". Mechanical Sciences, 2, pp. 119-127.

[11] Bachta, W., Renaud, P., Laroche, E., and Gangloff, J., 2009. "Cardiolock2: Parallel singularities for the design of an active heart stabilizer". In ICRA, pp. 3839-3844.

[12] Ramadan, A., Arai, T., Takubo, T., and Inoue, K., 2008. "Optimization of a hybrid two-fingered micro hand using genetic algorithms”. pp. $103-107$.

[13] Kicinger, R., Arciszewski, T., and Jong, K. D., 2005. "Evolutionary computation and structural design: A survey of the state-of-the-art". Computers \& Structures, 83(23-24), pp. $1943-1978$.

[14] Kaveh, A., Hassani, B., Shojaee, S., and Tavakkoli, S., 2008. "Structural topology optimization using ant colony methodology". Engineering Structures, 30(9), pp. 2559 2565.

[15] Luh, G.-C., and Lin, C.-Y., 2009. "Structural topology optimization using ant colony optimization algorithm". Applied Soft Computing, 9(4), pp. 1343 - 1353.

[16] Lobontiu, N., 2003. Compliant Mechanisms - design of 
flexure hinges. CRC Press.

[17] Yong, Y. K., Lu, T.-F., and Handley, D. C., 2008. "Review of circular flexure hinge design equations and derivation of empirical formulations". Precision Engineering, 32(2), pp. $63-70$.

[18] Dorigo, M., and Gambardella, L. M., 1997. "Ant colonies for the travelling salesman problem". Biosystems, 43(2), pp. $73-81$.

[19] Socha, K., and Dorigo, M., 2008. "Ant colony optimization for continuous domains". European Journal of Operational Research, 185(3), pp. 1155 - 1173.

[20] Blum, C., 2005. "Ant colony optimization: Introduction and recent trends". Physics of Life Reviews, 2(4), pp. 353 373.

[21] Bachta, W., Renaud, P., Laroche, E., Forgione, A., and Gangloff, J., 2008. "Cardiolock: an active cardiac stabilizer, first in vivo experiments using a new robotized device". Computer Aided Surgery, 13(5), pp. 243-254.

[22] Bachta, W., Renaud, P., Laroche, E., and Gangloff, J., 2011. "The cardiolock project: Design of an active cardiac stabilizer for cardiac surgery". ASME Journal of Mechanical Design, 133(7), july, pp. 071002-1-071002-10.

[23] Su, X.-P. S., and Yang, H. S., 2001. "Design of compliant microleverage mechanisms". Sensors and Actuators A: Physical, 87(3), pp. 146 - 156.

[24] Jouaneh, M., and Yang, R., 2003. "Modeling of flexurehinge type lever mechanisms". Precision Engineering, 27(4), pp. $407-418$.

[25] Jiang, J., and Mockensturm, E., 2006. "A motion amplifier using an axially driven buckling beam: I. design and experiments". Nonlinear Dynamics, Volume 43, pp. 391-409.

[26] Liu, A.-Q., Li, J., Liu, Z., Lu, C., Zhang, X., and Wang, M., 2006. "Self-latched micromachined mechanism with large displacement ratio". Microelectromechanical Systems, 15(6), dec., pp. 1576-1585.

[27] CedratTechnologies, 2008. Cedrat Piezo Product Catalogue.

[28] Parkinson, M. B., Jensen, B. D., and Kurabayashi, K., 2001. "Design of compliant force and displacement amplification micro-mechanisms". In Proc. ASME 2001 Design Engineering Technical Conferences.

[29] Sitti, M., 2003. "Piezoelectrically actuated four-bar mechanism with two flexible links for micromechanical flying insect thorax". Mechatronics, IEEE/ASME Transactions on, 8(1), march, pp. $26-36$

[30] Liaw, H. C., and Shirinzadeh, B., 2008. "Robust generalised impedance control of piezo-actuated flexure-based four-bar mechanisms for micro/nano manipulation". Sensors and Actuators A: Physical, 148(2), pp. 443 - 453.

[31] Ma, H.-W., Yao, S.-M., Wang, L.-Q., and Zhong, Z., 2006. "Analysis of the displacement amplification ratio of bridgetype flexure hinge". Sensors and Actuators A: Physical,
132(2), pp. $730-736$.

[32] Juuti, J., Kords, K., Lonnakko, R., Moilanen, V.-P., and Leppvuori, S., 2005. "Mechanically amplified large displacement piezoelectric actuators". Sensors and Actuators A: Physical, 120(1), pp. 225 - 231.

[33] Choi, K.-B., Lee, J. J., and Hata, S., 2010. "A piezodriven compliant stage with double mechanical amplification mechanisms arranged in parallel". Sensors and Actuators A: Physical, 161(1-2), pp. 173 - 181.

[34] Claeyssen, F., Letty, R. L., Barillot, F., and Sosnicki, O., 2007. "Amplified piezoelectric actuators: Static \& dynamic applications". Ferroelectrics, 351, pp. 3-14.

[35] Ueda, J., Secord, T. W., and Asada, H. H., 2010. "Large effective-strain piezoelectric actuators using nested cellular architecture with exponential strain amplification mechanisms". Mechatronics, IEEE/ASME Transactions on, 15(5), pp. $1-13$.

[36] Tian, Y., Shirinzadeh, B., Zhang, D., and Alici, G., 2009. "Development and dynamic modelling of a flexure-based scott-russell mechanism for nano-manipulation". Mechanical Systems and Signal Processing, 23(3), pp. 957 - 978.

[37] Schotborgh, W. O., Kokkeler, F. G. M., Tragter, H., and van Houten, F. J. A. M., 2005. "Dimensionless design graphs for flexure elements and a comparison between three flexure elements". Precision Engineering, 29(1), Jan., pp. 4147.

[38] Pilkey, W. D., and Pilkey, D. F., 2008. Peterson's Stress Concentration Factors (3rd Edition). John Wiley \& Sons. 